\title{
Constrained-Design of Passive UHF RFID Sensor Antennas
}

\author{
Cecilia Occhiuzzi, Member, IEEE, and Gaetano Marrocco
}

\begin{abstract}
Passive UHF RFID tags may be used, beside labeling, to remotely observe the physical/chemical change of the tagged object, through modulation of their impedance and gain, thus acting as sensor antennas. The design of this new class of devices can be mastered by fully understanding the relationship between communication and sensing with the purpose to balance the maximization of the dynamic range of the response with the stability of the read distance. A new kind of communication/sensing nomogram permits to display both behaviors in a unitary way and to predict their physical limits, as well as to formalize a multi-parameter general-purpose optimization methodology. The procedure is demonstrated by application to the design of a strain-gauge tag and of a level-detector wireless sensor.
\end{abstract}

Index Terms-Optimization, RFID, sensor.

\section{INTRODUCTION}

T HE possibility to use passive tags as sensing devices of objects, people and environment is becoming one of the most interesting and promising application of passive UHF Radio Frequency Identification (RFID) technology. By properly loading the tag with sensitive materials [1] or by using the bare antenna as a sensor of the surrounding environment [2], many different employments have been already envisaged and experimented, demonstrating the feasibility of the idea but, at the same time, the inadequacy of current antenna design methodologies [3].

The rationale of the tag-as-sensor is based on the fact that, since the tag's input impedance and gain depend on the nearby matter, any of its physical or chemical variation could affect directly (acting on the antenna) and indirectly (acting on the loading material) the tag's performance and be remotely detected by the reader. The generality of this basic principle allows in theory any passive tag to be used as a sensor of "effective" complex permittivity changes. However, to be successfully employed as sensors, this class of devices should be able to properly track the evolution of the phenomenon under observation, being for example monotonic, single-valued and sensitive enough at least in the most critical ranges.

Up to now, the published attempts to design sensing-oriented tags have not been focused on mastering the sensing response

Manuscript received August 06, 2012; revised November 29, 2012; accepted February 24, 2013. Date of publication March 07, 2013; date of current version May 29, 2013. This work was supported in part by project PRIN-2008: MULTI-TAG funded by Italian Ministry of University.

The authors are with the DISP, University of Roma Tor Vergata, 00133 Roma, Italy (e-mail: occhiuzzi@radio6ense.com; marrocco@disp.uniroma2.it).

Digital Object Identifier 10.1109/TAP.2013.2250473 which is presented as a side effect of that specific tag rather than a controllable variable.

RFID communication and sensing capabilities demand for opposite requirements. The tag's antenna is usually designed to be perfectly matched to the RFID IC in a reference condition, e.g., at the beginning of the phenomenon to be monitored, and it undergoes mismatching along with the process due to the variation of the input impedance [4]. A worsening of the power scavenging performances and in particular of the read distance is thus experienced and it is as worse as the sensitivity of the sensor is more relevant. Common critical issues of this class of radio-sensors are hence the trade-off between sensitivity [5] and communication performances [6]: in the first case the real usefulness of the radio-sensor is in question due to the poor sensing capabilities, in the second case the usability itself may become infeasible due to the impossibility to read the tag during the whole evolution of the process.

Since there is no decoupling from the operative and structural point of view between antenna and sensor, the traditional design procedures [7], mostly communication-oriented, have thus to be revisited or even rethought in order to handle both sensing and communication needs at the same time.

A first attempt to jointly optimize sensing and communication features of an RFID sensor tag can be found in [8]. The sensitivity can be enhanced by using high impedance ICs, while the inversion curves, relating the expected tag response to the change of the physical phenomena, may be shaped by a wise selection of the impedance matching condition. Although that paper proposes preliminary design guidelines, the method is mainly reduced to an iterative search for one status of the process where maximizing the power scavenged by the IC.

A preliminary idea about how handling in a unitary way the trade-off between communication and sensing requirements has been recently presented by the authors in [9]. Now, a more general and complete formulation enables a full shaping of the response of the Sensor Tag (S-tag) with an intrinsic measure of the quality of the design itself. At this aim, a convenient nomogram is here introduced with the purpose to understand the ultimate relationship between sensing and communication, and in particular to estimate the best achievable sensing capability that is compatible with the constraint over communication and power issues. This representation moreover helps to setup a multi-parameter iterative optimization, of general application, that basically solves a complex impedance matching problem. The potentiality of such a method is finally discussed by revisiting two examples taken from recent literature concerning the RFID sensing of the filling level of containers and of mechanical deformations. 


\section{SENSing AND COMmUNICATION CONSTRAINTS}

\section{A. Representations}

Let $\Psi(t)$ denote a local physical, chemical or geometrical parameter of the environment surrounding the tag which has to be monitored by the RFID reader. The RFID communication exploits a two-way link: a direct one, wherein the key-issue is the scavenging of power at the chip's port so that the tag may activate and perform actions, and a reverse link wherein the tag transmits its data to the reader by changing its reflectivity through impedance modulation. The link [10] for UHF-RFID systems (overall World-wide band: 866-956 MHz) is commonly characterized under the far field assumption by using the Friis formula for the direct path (1) so that the power $P_{R \rightarrow T}[\Psi]$ scavenged by the tag from the field coming from the reader can be expressed in terms of gain, polarization, impedance parameters and, of course, of the evolving process $\Psi$.

$$
P_{R \rightarrow T}[\Psi]=\left(\frac{\lambda_{0}}{4 \pi d}\right)^{2} P_{\mathrm{in}} G_{R}(\theta, \phi) G_{T}(\theta, \phi)[\Psi] \cdot \tau[\Psi] \eta_{p}
$$

where $d$ is the reader-tag distance, $G_{R}$ is the gain of the reader antenna, $G_{T}$ is the gain of the tag's antenna. $P_{\text {in }}$ is the power entering the reader's antenna, $\eta_{p}$ is the polarization mismatch between the reader and the tag, and $\tau$ is the power transmission coefficient of the tag:

$$
\tau[\Psi]=\frac{4 R_{\text {chip }} R_{A}[\Psi]}{\left|Z_{\text {chip }}+Z_{A}[\Psi]\right|^{2}}
$$

with $Z_{\text {chip }}$ input impedance of the RFID integrated circuit (IC) and $Z_{A}$ input impedance of the antenna.

The reverse link is instead characterized by the backscattered power emerging from the tag and hence collected by the reader

$$
P_{R \leftarrow T}[\Psi]=\frac{1}{4 \pi}\left(\frac{\lambda_{0}}{4 \pi d^{2}}\right)^{2} P_{\text {in }} G_{R}^{2}(\theta, \phi) \eta_{p}^{2} r c s_{T}(\theta, \phi)[\Psi]
$$

where $r c s_{T}$ is the tag's radar cross-section

$$
\operatorname{rcs}_{T}[\Psi]=\frac{\lambda_{0}^{2}}{4 \pi} G_{T}^{2}[\theta, \phi, \Psi(t)] \tau[\Psi] \frac{R_{a}[\Psi]}{R_{c h i p}}
$$

The backscattered power $P_{R \leftarrow T}$ is directly measurable by the reader [11] in terms of the Received Signal Strength Indicator (RSSI). RFID signaling requires the internal chip impedance to be switched between two impedances $Z^{O N}, Z^{O F F}$ originating a differential r.c.s $s_{T}$. [12]. The values of above impedances depend on the specific microchip and sensing information can be in principle gathered even by the differential r.c.s. itself [13]. For the sake of simplicity, the proposed methodology will be related to the very common choice of $Z^{O N}$ equal to the chip's impedance in the scavenging mode $\left(Z^{O N}=Z_{\text {chip }}\right)$ and $Z^{O F F} \gg Z^{O N}$ (at limit, an open circuit). In this case the differential r.c.s.T, and hence the received RSSI, will correspond to the "ON" state only ([13], [14]). The extension of the design method to a different kind of impedance modulation is straightforward.

Another parameter that can be measured by the reader is the turn-on power $P_{\text {in }}^{\text {to }}[\Psi]$, e.g., the minimum input power $P_{\text {in }}$ through the reader's antenna forcing the tag to respond. It can be derived from (1) by considering $P_{R \rightarrow T}=P_{c h i p}$, with the latter being the microchip sensitivity:

$$
P_{\text {in }}^{t o}[\Psi]=\left(\frac{\lambda_{0}}{4 \pi d}\right)^{-2} \frac{P_{\text {chip }}}{G_{R}(\theta, \phi) \eta_{p} G_{T}(\theta, \phi)[\Psi] \cdot \tau[\Psi]}
$$

Finally, forward (1) and backward (3) powers may be combined at turn-on, e.g., when the power collected by the tag $P_{R \rightarrow T}(\Psi)$ equals the IC sensitivity $P_{c h i p}$, with the purpose to drop out the influence of the distance and of the reader's and tag's gains and orientation. A non-dimensional indicator, denoted as Analog Identifier (AID), can be hence introduced as in [15]. According to the considered impedance modulation, the AID assumes the simple form:

$$
A I D[\Psi] \triangleq \frac{P_{\text {chip }}}{\sqrt{P_{R \leftarrow T}[\Psi] \cdot P_{\text {in }}^{t o}[\Psi]}}=\sqrt{\tau[\Psi] \frac{R_{\text {chip }}}{R_{a}[\Psi]}}
$$

In case of different modulations, above expression is modified just for a multiplying factor depending on $\left\{Z^{O N}, Z^{O F F}\right\}$ but still unaffected by reader-tag mutual position [15].

AID is a true signature of the tag's impedance and it is immune from the variability of the measurement conditions. Thus, it is useful to sense the variation of the objects in case of non-stationary sensing [3], for instance when hand-held devices are used, so that the reader-tag mutual position may be changed in successive measurements. Furthermore, as experimentally verified in [16], the combined use of direct and inverse-link powers permits to cancel the effect of the ambient multi-path, so that the detected variation of the AID can be univocally related to the change of the physical parameters under observation. The accuracy and the uncertainty of the considered sensing indicators have been deeply investigated in [17] and AID revealed a more robust and stable sensing-oriented indicator when compared with the standalone turn-on power and RSSI.

\section{B. Constraints}

Generally, the bottleneck of the whole tag-reader communication is imposed by the direct link, due to the rough chip power sensitivity (currently of the order of $10^{-5} \mathrm{~W}$ ) and by the maximum reader's power allowed by local regulations, which hence limits the maximum read range. The parameter that describes the tag's scavenging capabilities is definitely the realized gain $G_{\tau}=G_{T} \tau$, e.g the gain of the tag's antenna scaled by the mismatch to the RFID IC. Sensing capabilities are generally achieved at the expenses of read-distance degradation since the change of physical/chemical features of the environment are sensed by the tag through a deviation from its static gain and/or impedance matching. Hence, the true effectiveness of an S-tag results from the trade-off between sensing and communication.

Denoting with $\xi=\left\{A I D, P_{R \leftarrow T}, P_{\text {in }}^{\text {to }}\right\}$ any of the observable parameters of the RFID link, a trade-off solution to the sensing-communication problem is to observe the state of the object $\Psi(\mathrm{t})$ through a measurable quantity whose variation is not necessarily associated to a worsening of the communication performance. Such an indicator should be optimally a parameter of the reverse-link such as the backscattered power, which is however orientation-and position-dependent, or the Analog IDentifier $(A I D)$ if some degradation in the read distance can 
be accepted. The design procedure proposed in this paper will address the only AID indicator since, as shown later on, the combined control of communication and sensing may be conveniently reduced to considerations over the bare impedances.

As for usual sensors, the response of the S-tag can be quantified by the dynamic range, e.g., by the overall change of the measured parameters between the extreme interesting realizations of the process, $\Psi_{\min }, \Psi_{\max }$, conveniently expressed in decibel

$$
\Delta A I D=\left|A I D\left[\Psi_{\max }\right]-A I D\left[\Psi_{\min }\right]\right|_{d B}
$$

Sensing requirement induces the constraint

$$
\triangle A I D \geq R
$$

where $R$ is dependent on the reader resolution $\delta A I D$ and on the desired number $n=R / \delta A I D$ of observable distinct states of the process between the extreme detectable values. It is moreover requested that $A I D(\Psi)$ is monotonic with respect to $\Psi$.

Preserving a useful read range in all the states of the process means to enforce a condition over the minimum turn-on power

$$
P_{\text {in }}^{t o}(\Psi) \leq p_{\text {max }} \text { for } \Psi_{\text {min }} \leq \Psi \leq \Psi_{\max }
$$

The bound $p_{\max }$ has to comply with the local power regulations and with the true available power for fixed or hand-held readers. Having selected the reader-tag distance $d_{0}$, such a constrain will in turn enforce, from (5), the minimum allowed value for the realized gain, e.g.

$$
G_{T}(\Psi) \tau(\Psi) \geq g \text { for } \Psi_{\min } \leq \Psi \leq \Psi_{\max }
$$

where $g$ is given by (9) and (5):

$$
g=\frac{P_{c h i p}}{p_{\max } G_{R} \eta_{p}}\left(\frac{4 \pi d_{0}}{\lambda_{0}}\right)^{2}
$$

It is worth noticing that real-world applications have to face with multi-path environments so that the direct link relationship is generally more complex than (5). However, as discussed in [18], correction coefficients may be included in the communication constraint in order to account for main interactions such as that generated by adjacent walls.

\section{COMmUNiCATION-SENSING NOMOgRAm}

For the sake of clarity, it will be hereafter assumed that the variation of the tag's gain with respect to the process in evolution is weaker than the variation of the power transfer coefficient, so that $(d / d \Psi)\left(G_{T} \tau\right) \simeq G_{T}\left(\Psi_{0}\right)(d / d \Psi) \tau$, where $\Psi_{0}$ is a realization of the process. This is true in case of some deformable meander line antennas used as strain-gauge [19] or when mechanical sensors are embedded, such as shape memory alloys [20] or shape memory polymers [21]. This hypothesis will be however removed further on by introducing a general purpose iterative design procedure.

Under this assumption, the communication and sensing features of a tag may be conveniently referred to the parameters

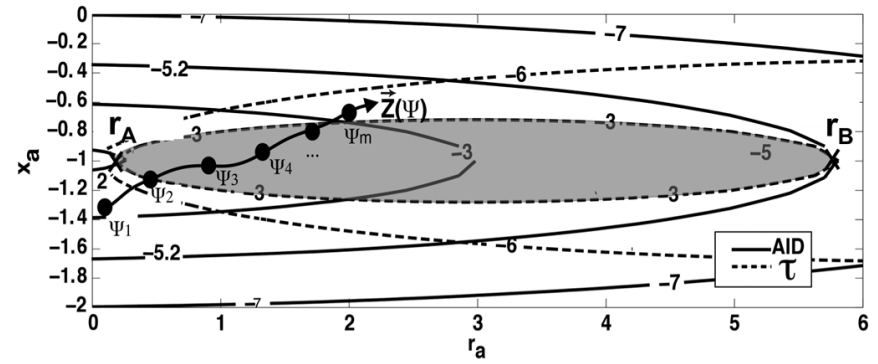

Fig. 1. Iso-line of constant AID and $\tau$ on the normalized antenna impedance plane, for $\mathrm{Q}=10$. In grey the area corresponding to $\tau_{\min }=-3 \mathrm{~dB}$. The curve $\vec{Z}[\Psi]=r_{a}(\Psi) \hat{i}+x_{a}(\Psi) \hat{j}$ indicates a possible variation of the antenna's impedance all along the process in evolution.

$\{\tau[\Psi], A I D[\Psi]\}$ which only depends on impedance change of the antenna. To visually represent the relationship between the communication $(\tau)$ and sensing (AID) parameters, a particular kind of nomogram is now introduced. This will be useful to estimate the theoretical maximum dynamic range of the tag that can be achievable within the communication constraints in (8) and (10) and moreover, to set up a design procedure. At this purpose, power transmission coefficient and Analog Identifier could be expressed in terms of normalized input impedance of the tag $r_{a}=R_{A} / R_{\text {chip }}, x_{a}=X_{a} / X_{\text {chip }}$, with $Q=\left|X_{\text {chip }}\right| / R_{\text {chip }}$ the quality factor of the chip:

$$
\begin{aligned}
\tau[\Psi] & =\frac{4 r_{a}}{\left|1+r_{a}+j Q\left(1+x_{a}\right)\right|^{2}} \\
\operatorname{AID}[\Psi] & =\frac{2}{\left|1+r_{a}+j Q\left(1+x_{a}\right)\right|}=\sqrt{\frac{\tau}{r_{a}}}
\end{aligned}
$$

Having fixed $Q$, a chart of $\{\tau, \operatorname{AID}\}$ isolines is produced in decibel by varying $r_{a}$ and $x_{a}$ (Fig. 1). Such lines are ellipses with major axis over $x_{a}=-1$ line and whose eccentricity depends on the quality factor $Q$ of the microchip. In particular, ellipses become narrower (more oblate on the $x$-axis) [22] as the quality factor $\mathrm{Q}$ increases. The point $\left(r_{a}=1, x_{a}=-1\right)$ corresponds to the matched state $\Psi_{M}$ [8], e.g., the state of the process for which the tag's antenna shows the best matching to the IC: there, $Z_{a}=Z_{\text {chip }}^{*}$ and AID $=\tau=0 \mathrm{~dB}$.

The $\tau$-AID nomogram does not depend on the particular antenna layout. The variation of the tag's impedance all along the phenomenon evolution can be therefore traced over such a plane by a sequence of couplets $\left\{r_{a}\left(\Psi_{n}\right), x_{a}\left(\Psi_{n}\right)\right\}$ describing an oriented curve (Fig. 1):

$$
\vec{Z}[\Psi]=r_{a}(\Psi) \hat{i}+x_{a}(\Psi) \hat{j} \in \mathbb{R}^{2}
$$

According to the intercepted isolines, the radio sensor will show different sensing and communication behavior all along the process. The sensing response $A I D(\Psi)$ will be monotonic if the impedance curve $\vec{Z}[\Psi]$ does not intersect any AID isoline in more than a point, which means that $\vec{Z}[\Psi]$ can not draw loops or arcs.

Communication constrain in (9) or in (10) becomes a condition over the power transfer coefficient

$$
\tau[\Psi] \geq \frac{g}{G_{T}\left(\Psi_{0}\right)} \equiv \tau_{\min }
$$


Such a condition defines the region (shadowed in Fig. 1) wherein the allowed dynamic range of the tag response has to be constrained. Thus, the maximum variation $\triangle \boldsymbol{A I D}$ will be given by the AID isolines passed by the extremes $A=\left(r_{A},-1\right)$, $B=\left(r_{B},-1\right)$ of the major axis of the $\tau=\tau_{\min }$ ellipse, where $r_{A}$ and $r_{B}$ are deduced from (2) by solving for $r_{a}$ :

$$
r_{A, B}=\left(-1+\frac{2}{\tau_{\min }} \mp 2 \sqrt{\frac{1}{\tau_{\min }^{2}}-\frac{1}{\tau_{\min }}}\right)
$$

Thus, the path $\vec{Z}[\Psi]$ which optimizes the sensing dynamic range is a straight segment $\vec{Z}[\Psi]=A \leftrightarrow B$ on the $r_{a}$-axis, which means that the best sensing mechanism over the antenna is such to convert the variation of the process into a change of the only input resistance. As a consequence, resistive sensing mechanisms are more effective than capacitive or mixed ones.

The theoretical upper bound of the sensing range that is compatible with the constraint over $\tau$, e.g., corresponding to the $\mathrm{AB}$ segment, is now easily obtained by evaluation of (13) for $x_{a}=-1$ and $r_{A, B}$, e.g.

$$
\begin{aligned}
\Delta A I D_{\max } & =A I D(B)-A I D(A) \\
& =10 \log \left(\frac{1+r_{B}}{1+r_{A}}\right) \\
& =10 \log \left(\frac{1+\sqrt{1-\tau_{\min }}}{1-\sqrt{1-\tau_{\min }}}\right)
\end{aligned}
$$

and it is independent on the $Q$-factor of the chip. The iso- $\tau_{\min }$ curves with different $Q$ present indeed the same center, the same major axis $\left(r_{a}\right)$ while instead a different minor axis $\left(x_{a}\right)$ [22]. Since the maximum achievable sensing range is identified by the AID isolines passing through the extremes of the major axis $\tau=\tau_{\min }, \Delta A I D_{\max }$ is theoretically unaffected by the $\mathrm{Q}$ factor of the chip. However, in case of real configurations, when the impedance curve is not exactly kept onto the horizontal axis, the increase of the $\mathrm{Q}$ factor, and accordingly the narrowing of vertical axis of the gray ellipse in (Fig. 1), will reduce the observable dynamic range of the AID. Thus, low-Q microchips should be preferred for a communication-constrained sensing RFIDs.

The diagram $\Delta A I D_{\max }\left(\tau_{\min }\right)$ in Fig. 2 provides an operative tool to manage the most useful tradeoff between read-range degradation and sensing dynamic range. For instance the choice $\tau_{\min }=-3 \mathrm{~dB}$ will permit not to degrade the read range below the $70 \%$ of its maximum value corresponding, for example, to the initial (or final) state of the process. Accordingly, the theoretical maximum range of the tag's response will be $-5.2 \mathrm{~dB} \leq$ $A I D[\Psi] \leq 2.3 \mathrm{~dB}(\triangle A I D=7.5 \mathrm{~dB})$ as observed by the intersections between the isolines in Fig. 1.

\section{Optimization Procedure}

Previous Section demonstrates that, having fixed a constraint over the minimum read range, the maximum extent of the sensing dynamics could be achieved by shaping the profile of the antenna impedance v.s. the process evolution so that the normalized input impedance moves along the major axis of the shadowed ellipse in the nomogram of Fig. 1. Such a shaping can be achieved by acting onto an impedance adapter (T-match, Loop Match, Slot Match [23]) of a fixed radiating

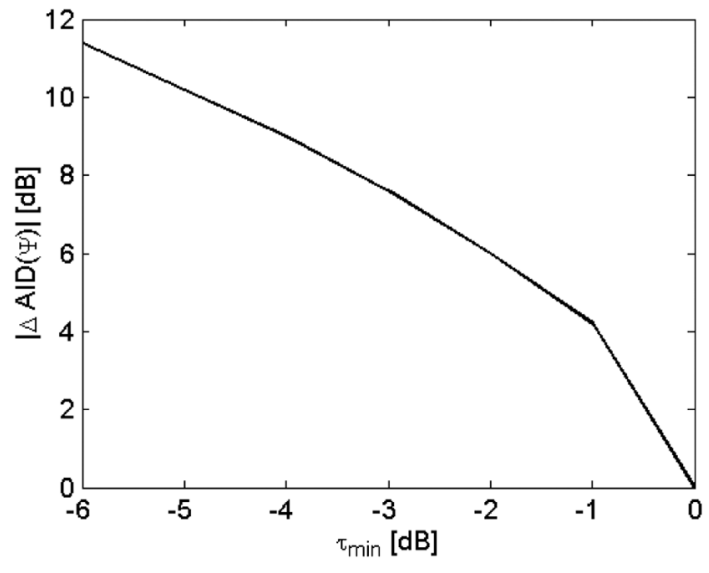

Fig. 2. Maximum effective sensing capabilities of the radio-sensor for constant-gain process.

body, or instead by controlling all the geometrical parameters of the tag, hereafter denoted as $\boldsymbol{\alpha}=\left\{\alpha_{1}, \alpha_{2} \ldots \alpha_{P}\right\}$. Hence, the design of the tag-as-sensor reduces to the minimization of the following penalty function

$$
\sum_{n=0}^{N}\left\|\vec{Z}\left(\Psi_{n}\right)[\boldsymbol{\alpha}]-\left(r_{A}+n \frac{\left|r_{A}-r_{B}\right|}{N}\right) \hat{i}+\hat{j}\right\|_{2} \rightarrow 0
$$

where $n=1 \ldots N$ indicates a convenient discretization of the process evolution.

The quality of the obtained tag as sensor, e.g., how close the achieved dynamic range is to the upper bound, can be definitely quantified by the sensor efficiency

$$
\eta_{S}=\frac{\Delta A I D(\boldsymbol{\alpha})}{\Delta A I D_{\max }} \leq 1
$$

Practical implementation of the tag design methodology is described next for the most general case of processes that produce a variation of both the input impedance and the gain of the tag. When the antenna is loaded by a resistive sensor [1] or when the surrounding environment produces a redistribution of the radiated field (tag used as indicator of liquids' level [8]) the gain can not be considered constant anymore as in previous Section. Accordingly all the communication and sensing indicators retain the combined dependance of $G_{T}[\Psi]$ and $\tau[\Psi]$, and in particular the constraint over the read distance needs to be modified to include also the lowest values of the tag's gain. The only hypothesis for application of the following procedure is that the variation of the tag's gain is monotonic with respect to the parameter $\Psi$. If not, the process' evolution may be split into monotonic portions each of them sensed by a specific tag.

The constrained design procedure (also described by a flowchart in Fig. 3) works as in the following:

1) Define the process $\Psi$ to be monitored, and its most relevant realizations $\left\{\Psi_{1}, \Psi_{2} \ldots \Psi_{N}\right\}$.

2) Define the communication constraint $g$ (11)

3) Define the antenna topology and the geometrical parameters $\boldsymbol{\alpha}$ to be optimized and the microchip family $\left(Z_{c h i p}\right)$.

4) Produce a first trial of antenna layout $\boldsymbol{\alpha}^{(\mathbf{0})}=$ $\left\{\alpha_{1}^{(0)}, \alpha_{2}^{(0)}, \ldots\right\}$ in order to achieve the conjugate matching in a particular state of the process, for instance at the starting point $\Psi_{1}$, e.g., so that $Z_{a}\left(\Psi_{1}\right)=Z_{\text {chip }}^{*}$. 


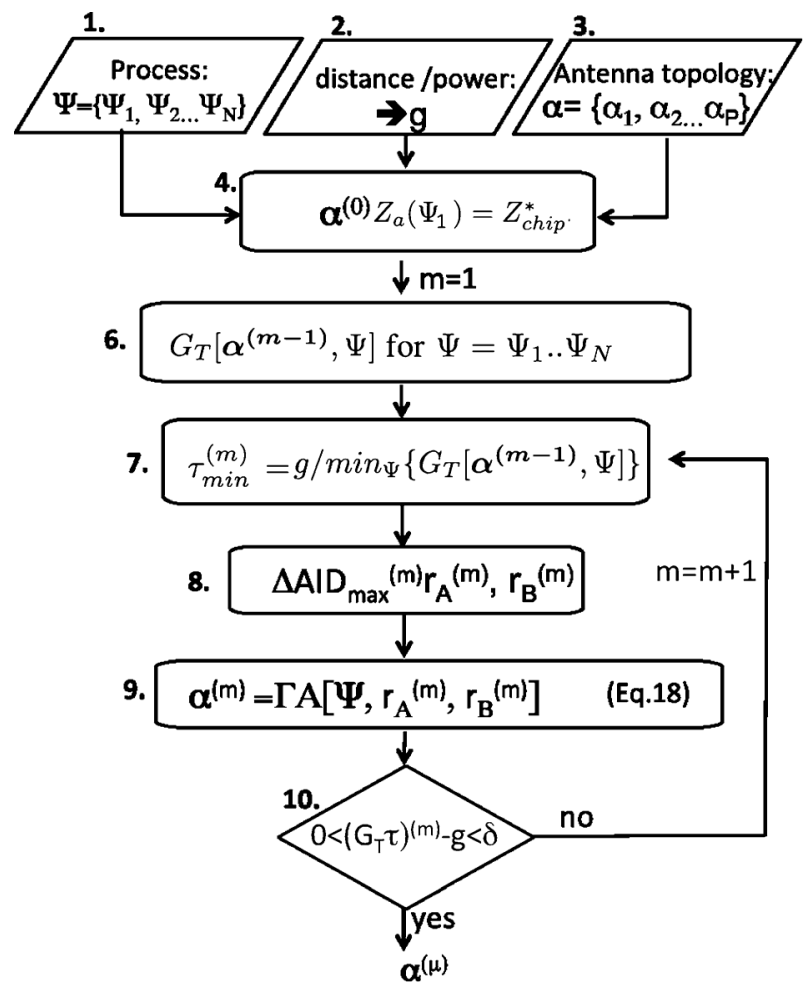

Fig. 3. Design Flow Chart for communication-constrained sensing tags.

5) set counter $m=1$

6) Calculate $G_{T}\left[\boldsymbol{\alpha}^{(\boldsymbol{m}-1)}, \Psi\right]$ for $\Psi=\Psi_{1} \ldots \Psi_{N}$

7) Set up the constraint $\tau_{\text {min }}^{(m)}=\mathrm{g} / \min _{\Psi}\left\{G_{T}\left[\boldsymbol{\alpha}^{(\boldsymbol{m}-\mathbf{1})}, \Psi\right]\right\}$ to preserve the requested communication performance even in the worst gain. Accordingly, the upper bound of the sensing dynamic range $\Delta A I D_{\max }$ is fixed by (17), or by the Fig. 2.

8) Evaluate from (16) the edge points $r_{A}\left(\tau_{m i n}^{(m)}\right), r_{B}\left(\tau_{m i n}^{(m)}\right)$ of the ideal $\vec{Z}(\Psi)$ function over the boundary ellipse $\tau=$ $\tau_{m i n}^{(m)}$ in the $\tau-A I D$ nomogram.

9) Solve the optimization problem in (18) for $\boldsymbol{\alpha}^{(\boldsymbol{m})}$

10) Check the condition $0 \leq\left(G_{T} \tau\right)\left[\boldsymbol{\alpha}^{(\boldsymbol{m})}, \Psi\right]-g \leq \delta$ for the actual antenna layout, with $\delta$ a small threshold.

11) If step 10 is verified, then exit, while if not,

12) $m=m+1$, go back to step 6 .

The condition in the step 10 has the meaning to take benefit from the gain of the antenna which would be in excess with respect to what required $(g)$ to establish the communication. In this case the constrain $\tau_{\min }$ can be relaxed down to a lower value and accordingly to Fig. 2 the maximum sensing range $\triangle A I D$ will be improved as it will shown in the example Section.

At the end of the iterative procedure, the resulting tag will exhibit the maximum sensing range $\triangle A I D$ which is compatible with the requirement over the reading distance. By representing the obtained impedance path $\vec{Z}[\Psi]$ over the $\tau-A I D$ nomogram it will be moreover possible to have a visual indication about how close the performance of the designed antenna is from the optimum theoretical one (e.g., $\vec{Z}[\Psi]$ should be a segment from point $\mathrm{A}$ to $\mathrm{B}$ ).

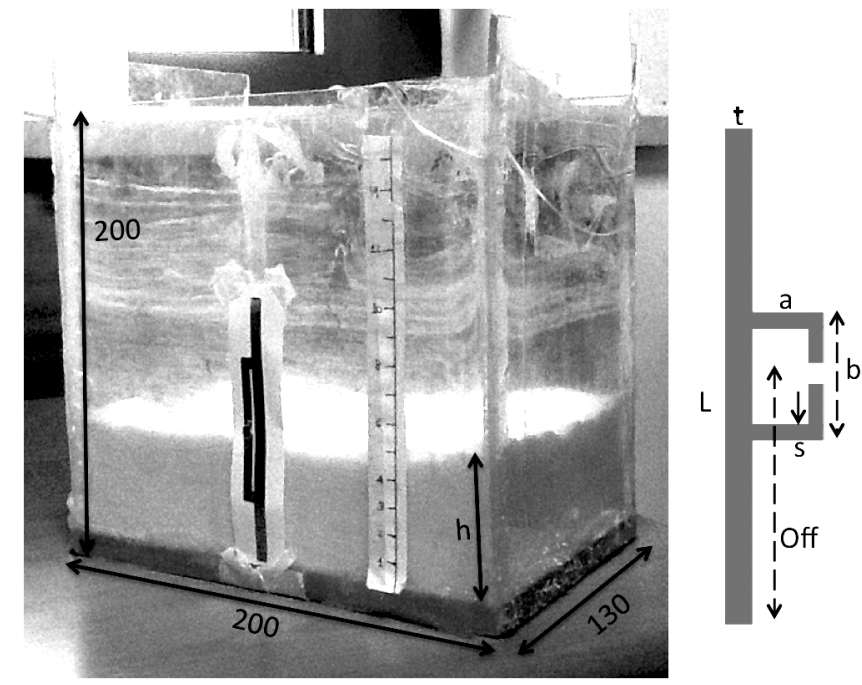

Fig. 4. Level sensor-A T-match dipole-like tag attached onto a perspex box containing sugar of variable level $h$. The geometrical parameter to be optimized are $\boldsymbol{\alpha}=\{a, b, s\}$, while fixed parameters are: (sizes are in [mm]) are $t=5$, Off $=45$ and $L=100$.

\section{EXAMPLES}

The proposed design methodology is now illustrated by application to a couple of realistic RFID radio-sensors useful to monitor the filling level of containers and the deformation of objects. Such examples are taken from recent papers where the devices have been deeply investigated both theoretical and experimentally, but without any effort to handle the combined communication-sensing problem.

Both examples are simulated by the Finite Difference Time Domain (FDTD) tool and the impedance discussion are referred to a typical chip of parameters $Z_{c h i p}=15-j 135 \Omega(\mathrm{Q}=9)$ and power threshold $P_{\text {chip }}=-15 \mathrm{dBm}$. For validation purpose, the first example has been finalized to a prototype fabrication and real-life measurements.

\section{A. Effective-Permittivity Sensor}

A bare tag was demonstrated in [24] to be able to sense the change of effective permittivity of the attached object by means of the variation of the input impedance and gain of the tag's antenna. The case-study geometry, e.g., a perspex container filled by $\operatorname{sugar}\left(\epsilon_{r}=2.76, \sigma_{r}=2.44 \cdot 10^{-2} \mathrm{~S} / \mathrm{m}\right)$, is reproduced in Fig. 4, and the filling level $h$ of the sugar is assumed to change as $0 \leq h \leq 11 \mathrm{~cm}$. The antenna topology is a half-wave dipole at $870 \mathrm{MHz}$ provided with a T-match adapter [23]. The set of geometrical parameters to be optimized is $\boldsymbol{\alpha}=\{a, b, s\}$. The width $s$ of the T-match plays in particular a considerable effect on the real part of the input impedance, since it determines the distribution of the electric current on the two main radiators.

The design goal is a read distance not less than $1 \mathrm{~m}$ by assuming a circular polarized reader's antenna $\left(\eta_{p}=0.5\right)$ emitting $E I R P=p_{\max } G_{R}=0.5 \mathrm{~W}$ (as in case of mobile devices). From (11) the realized gain of the tag will be therefore constrained such to include the minimum value of the gain, e.g., $\left(G_{T} \tau\right)[h] \geq g=-6 \mathrm{~dB}$. The iterative design procedure in Section IV is applied as follows (numbering referred to flowchart in Fig. 3): 


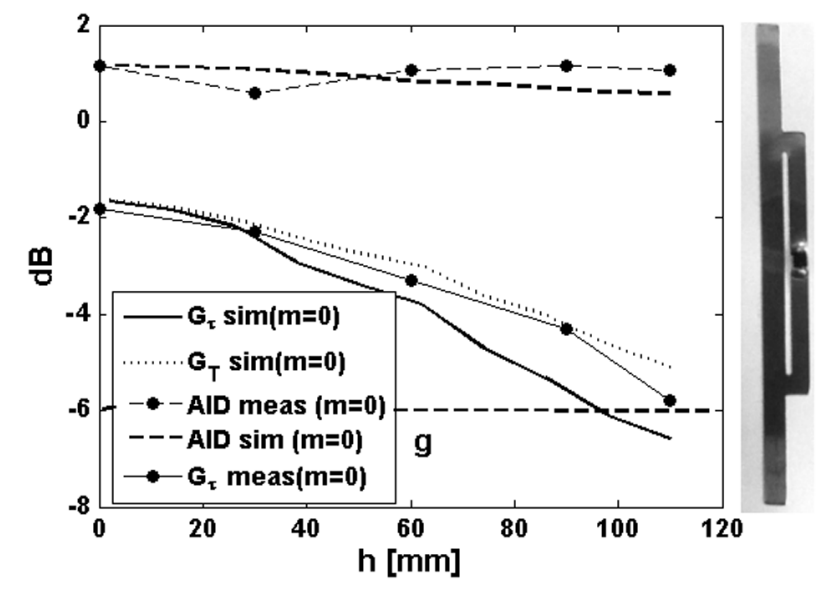

Fig. 5. Level Sensor-numerically estimated $A I D^{(0)}[h]$, gain $G_{T}^{(0)}[h]$ and realized $G_{\tau}^{(0)}[h]$ of the zero-order antenna layout $\alpha^{(0)}$ as corresponding to the change of the sugar level. Superimposed, measured $A I D^{(0)}[h]$ and $G_{\tau}^{(0)}[h]$ for the prototype shown on the right.

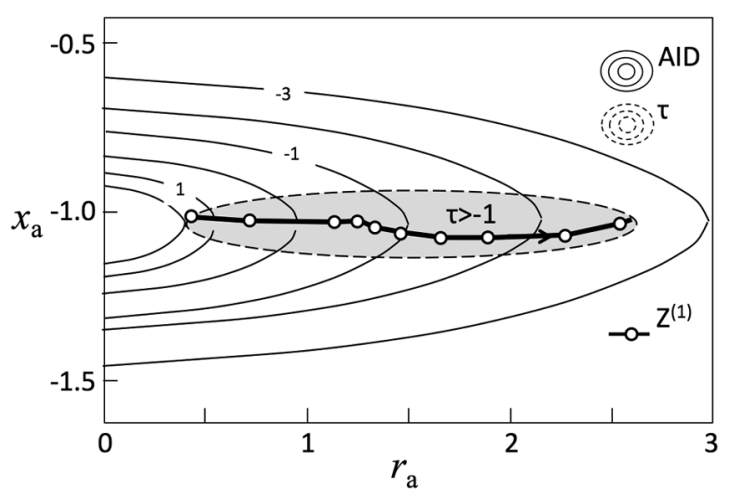

Fig. 6. Level sensor- $\{\tau, A I D\}$ chart (in dB) for the optimized tag with parameters $\alpha^{(1)}=\{10,28,5\} \mathrm{mm}$. The arrow indicates the verse of the process' evolution $0<h<11 \mathrm{~cm}$.

4) A first guess of the antenna layout is obtained by selecting the T-match parameters $\alpha^{(0)}=\{5,50,4\} \mathrm{mm}$ such to produce the best impedance matching between the antenna and the microchip in the reference condition of empty container $\left(\Psi_{M} \equiv\right.$ $h=0 \mathrm{~cm})$. This is an arbitrary choice and different conditions could be possible, for instance a half-filled of full-filled box.

6) Numerical simulations are applied to estimate the variation of the tag's gain (Fig. 5) produced by the change of the sugar level. The minimum value in the broadside direction is $\min \left\{G_{T}\left[\boldsymbol{\alpha}^{(\mathbf{0})}, h\right]\right\}=-5 \mathrm{~dB}$.

7) The power transfer coefficient is hence constrained as $\tau[h] \geq \mathrm{g} / \min \left\{G_{T}\left[\boldsymbol{\alpha}^{(\mathbf{0})}, h\right]\right\}=-6 \mathrm{~dB}-(-5 \mathrm{~dB})=-1 \mathrm{~dB} \equiv$ $\tau_{\min }^{(1)}$ with the purpose to preserve the minimum required distance also in the worst case. The theoretical upper bound for the AID variation will be accordingly $\Delta A I D_{\max }=4 \mathrm{~dB}$, as deduced from Fig. 2 or from (17).

8) the parameters of the $(\tau=-1 \mathrm{~dB})$-ellipse are $r_{A}^{(1)}=0.37$, $r_{B}^{(1)}=2.65$;

9) the optimization problem in (18) is solved by the Genetic Algorithm yielding the first iteration $\alpha^{(1)}=\{10,28,5\} \mathrm{mm}$. The corresponding impedance curve $\vec{Z}^{(1)}[h]$ lays well inside the shadowed ellipse (Fig. 6) and mostly onto the horizontal axis as required for maximum dynamic range.

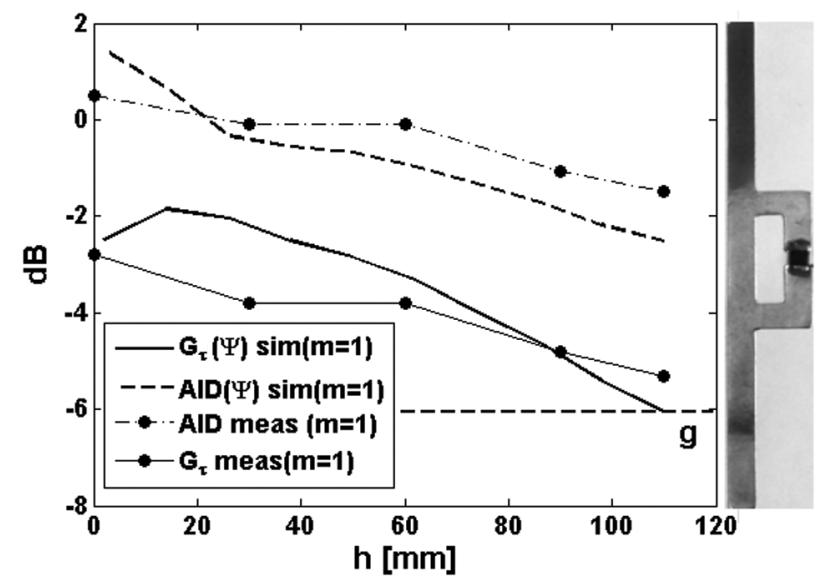

Fig. 7. Level sensor-Communication and sensing performances of the optimized filling level-sensor (prototype shown on the right) after the first iteration of the optimization procedure, measured and simulated values.

10) The variation of the tag's realized gain with respect to the sugar height (Fig. 7) is such that $\min \left\{\left(G_{T} \tau\right)\left[\boldsymbol{\alpha}^{(\mathbf{1})}, h\right]\right\}=$ $-6 \mathrm{~dB}=g$ therefore matching the original communication constraint.

No further optimization iteration is required and the actual layout $\boldsymbol{\alpha}^{(1)}$ is the final solution of the design problem. The achieved sensitivity range is $\triangle A I D=4 \mathrm{~dB}$, that is coincident with the theoretical upper bound (sensor efficiency $\eta_{S}=0 \mathrm{~dB}$ ). The obtained radio-sensor is therefore able to provide the optimum sensing performance permitted by the communication constraint.

To check the real-life feasibility of the optimization method, the two antennas resulting from step $m=0$ an step $m=1$ (see insets Fig. 5 and Fig. 7) have been fabricated by a copper sheet and the observation of sugar-filling experiment has been replicated by using a ThingMagic M5e reader.

Although there are some differences between measured and simulated data, reasonably due to the typical numerical tool's and instruments' uncertainties, the dynamic ranges of both sensors are in correct agreement with the data predicted by the method. In particular, the zero-order tag layout $\alpha^{(0)}$ shows a pretty constant $A I D^{(0)}[h]$ and a $\Delta G_{\tau}^{(0)}[h]=4 \mathrm{~dB}$, while the optimized first-order tag $\alpha^{(1)}$ offers a $\Delta A I D^{(1)}[h] \approx 3 \mathrm{~dB}$ with a relatively small variation of the communication capability $\Delta G_{\tau}^{(1)}[h]=3 \mathrm{~dB}$.

\section{B. Strain-Gauge Tag}

A deformable meander line antenna (MLA) with a T-match (Fig. 8), can be used as a passive strain sensor [19] for deformations occurring along its main length $h$. The dynamic range and sensitivity can be in principle adjusted by acting onto the $\mathrm{T}$-match form factor.

In the present example, the process to be monitored is a linear deformation of the antenna that is described by the strain $\epsilon=$ $\Delta h / h \cdot 100$, from a quite state $\epsilon_{1}=0 \%$ to a stretched state $\epsilon_{N}=30 \%$. The exploited antenna layout is a meandered dipole, provided with a T-match adapter. To find the optimum compromise between the read-range performance and the sensing dynamic range, the optimization procedure is applied to the geometrical parameters $\boldsymbol{\alpha}=\left\{h_{0}, b, N, w, h_{1}\right\}$ of the tag, e.g., to 

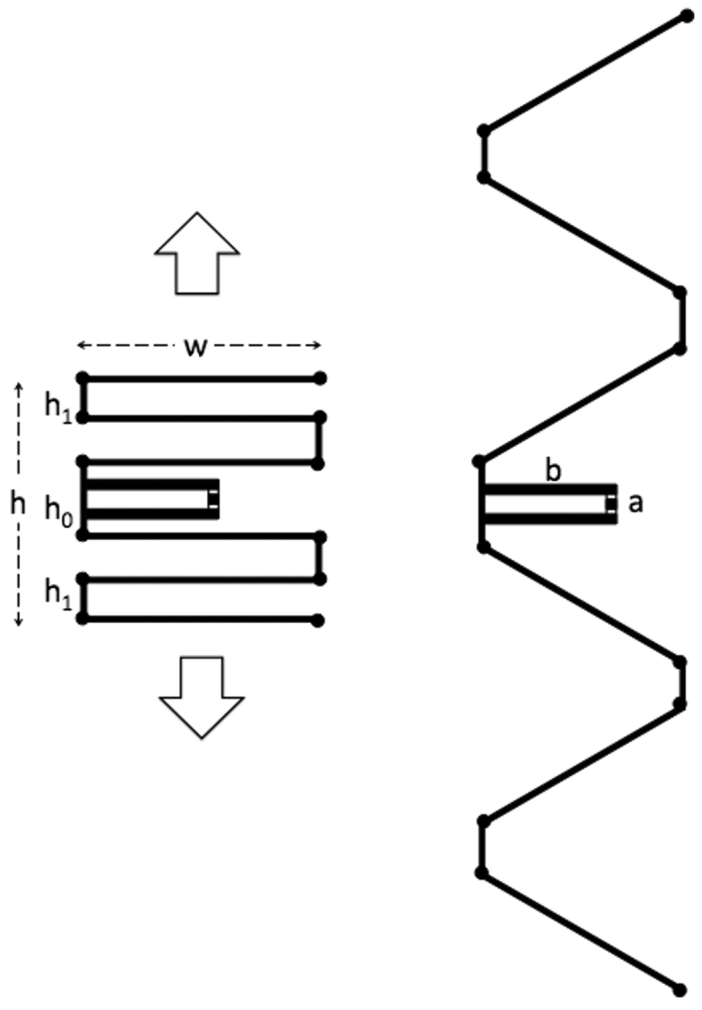

Fig. 8. Strain Sensor-meandered tag layout. Left) Quite State; Right) Stretched State. The geometrical parameters to be optimized are $\alpha=$ $\left\{h_{0}, b, N, w, h_{1}\right\}$, with $\mathrm{N}$ number of turns for each half structure.

the shape factor of the T-match as in the previously cited paper, and to the meandering profile. The design idea is to reduce the variation of the input reactance, mainly due to the mutual distance between the facing segments, and to enhance the variation of the input resistance, affected instead by the overall antenna's length $h$.

The design goal is a read distance not less than $2 \mathrm{~m}$ by assuming a circular polarized reader's antenna $\left(\eta_{p}=0.5\right)$ emitting $E I R P=p_{\max } G_{R}=0.5 \mathrm{~W}$ in any state of the deformation process. From (11) the realized gain of the tag will be therefore constrained such to include the minimum value of the gain, e.g., $\left(G_{T} \tau\right)[\epsilon] \geq g=-1 \mathrm{~dB}$. The iterative design procedure in Section IV is applied as follows:

4) the 0 th order guess of the antenna layout is obtained from [19], by considering $\alpha^{(0)}=\{6,18,1,36,7\}$. Such a tag shows the best matching $(\tau=0.9)$ between antenna and IC for $\epsilon=$ $5 \%$.

6) As in [19], the gain of the tag reveals weakly dependent on the considered deformations, with a minimum value $\min \left\{G_{T}\left[\alpha^{(0)}, \epsilon\right]\right\}=0.4 \mathrm{~dB}$.

7) The power transfer coefficient is hence constrained as $\tau[\epsilon] \geq \mathrm{g} / \min \left\{G_{T}\left[\alpha^{(0)}, \epsilon\right]\right\}=-1 \mathrm{~dB}-(0.4 \mathrm{~dB})=-1.4 \mathrm{~dB}$. A $\tau_{\min }=-1 \mathrm{~dB}$ is hence fixed with the purpose to preserve the minimum required distance also in the worst case. The theoretical upper bound for the AID variation will be accordingly $\Delta A I D_{\max }=4 \mathrm{~dB}$, as deduced from Fig. 2 or from (17).

8) the parameters of the $(\tau=-1 \mathrm{~dB})$-ellipse are again $r_{A}^{(1)}=$ $0.37, r_{B}^{(1)}=2.65$ as in the previous example since such parameters do not depend on the particular shape of the antenna;

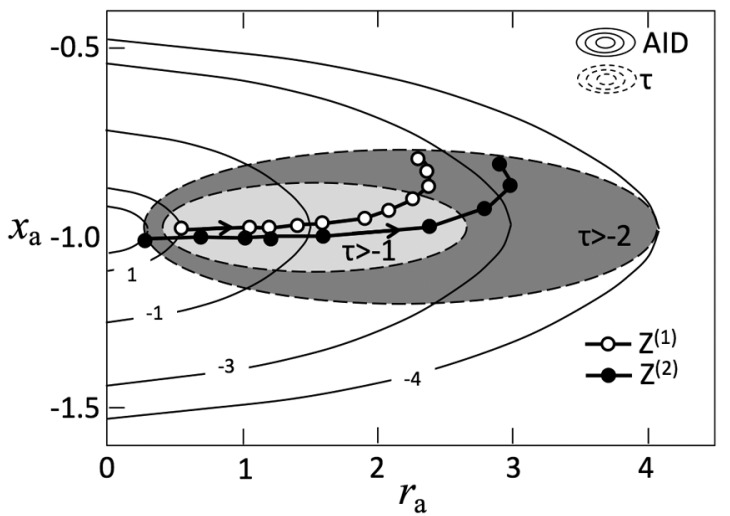

Fig. 9. Strain sensor- $\{\tau, A I D\}$ chart (in dB). Impedance curves $\overrightarrow{Z^{(1)}}$ and $\overrightarrow{Z^{(2)}}$ of the meandered tag in the first two iterations of the design procedure; the arrows indicate the verse of the process' evolution $0<\epsilon<30 \%$. The resulting dynamic ranges of the response are $\Delta A I D^{(1)}=3 \mathrm{~dB}$ and $\Delta A I D^{(2)}=5 \mathrm{~dB}$ respectively.

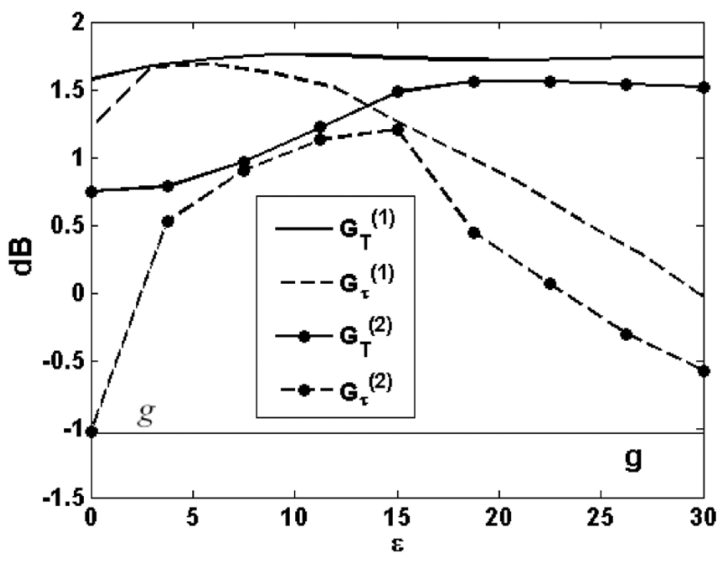

Fig. 10. Strain sensor-numerically estimated gain $G_{T}^{(m)}[h]$ and realized $G_{\tau}^{(m)}[h]$ in the first two iterations of the design.

9) the optimization problem in (18) is solved by the Genetic Algorithm yielding the first iteration $\alpha^{(1)}=$ $\{9,21,1,20,20\} \mathrm{mm}$. The corresponding impedance curve $\vec{Z}^{(1)}[\epsilon]$ lays mostly inside the shadowed $(\tau=-1 \mathrm{~dB})$ ellipse (Fig. 9) but it sensibly deviates from the horizontal axis in the second half of the process' evolution;

$10)$ the achieved AID dynamic range is $\Delta A I D^{(1)}[h]=3 \mathrm{~dB}$ and the variation of the tag's realized gain (Fig. 10) along with the deformation is such that $\min \left\{\left(G_{T} \tau\right)\left[\alpha^{(1)}, \epsilon\right]\right\}=0 \mathrm{~dB}>g$. It is hence possible to benefit from the $1 \mathrm{~dB}$ gain excess with respect to the constrain $g=-1 \mathrm{~dB}$ and tolerate a less rigid condition for the power transfer coefficient, e.g., $\tau_{\min } \rightarrow-2 \mathrm{~dB}$. From Fig. 2, this choice will enable an higher theoretical upper bound $\triangle A I D_{\max } \rightarrow 6 \mathrm{~dB}$.

11) the new parameter of the $(\tau=-2 \mathrm{~dB})$-ellipse are $r_{A}^{(2)}=$ $0.24, r_{B}^{(2)}=4.1$

12) the optimization problem in (18) is again solved by GA yielding the second iteration $\alpha^{(2)}=\{8,26,1,15,25\} \mathrm{mm}$. The corresponding impedance curve $\vec{Z}^{(2)}[h]$ is now fully inside the new shadowed ellipse (Fig. 6) and is flatter than in the previous iteration, hence giving an improved dynamic range.

13) the variation of the tag's realized gain (Fig. 10) with respect to the filling level is such that $\min \left\{\left(G_{T} \tau\right)\left[\alpha^{(2)}, h\right]\right\}=$ 


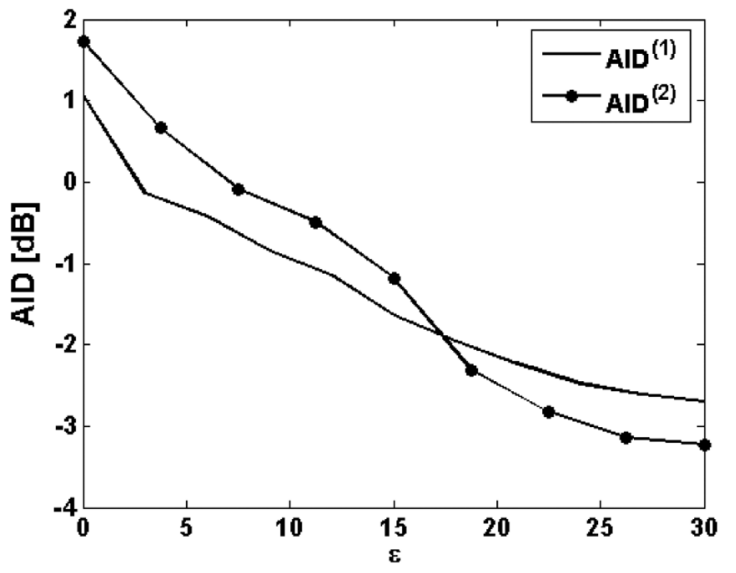

Fig. 11. Strain sensor-Variation of the tag's AID in the first two iterations of the design procedure.

$-1 \mathrm{~dB}=g$ therefore now matching the required communication constraint.

No further optimization iteration is required and the actual layout $\boldsymbol{\alpha}^{(2)}$ is the final solution of the design problem. The overall dynamic range of the Analog Identifier is $\triangle A I D^{(2)}=$ $5 \mathrm{~dB}$ which is however $1 \mathrm{~dB}$ narrower than the theoretical upper bound and the corresponding sensor efficiency is $\eta_{S}=-1 \mathrm{~dB}$.

\section{CONCLUSION}

The design of RFID radio sensors can be fully addressed by jointly considering communication and sensing requirements. Having defined the maximum acceptable degradation of the communication performance, the best sensing capabilities are mathematically defined by the natural relationship between the direct and reverse RFID links, regardless the antenna layout or the kind of process under observation. The optimization can be guided by using an innovative nomogram able to represent on a unique plane the communication-sensing behavior of a tag and also to provide indication about the upper bound of the achievable performances. A remarkable result is that the most effective way for an RFID antenna to "sense" the physical status of an object, with negligible degradation of communication, is to convert the change of the external phenomenon into a variation of the only input resistance, while preserving the reactance as stable as possible.

Although the proposed procedure is of general application and provides a first handy methodology to engineering the passive sensing by RFID, the choice of the antenna layout, as well as of the set of free geometrical parameters, are still a key point in approaching the best achievable trade-off between reading distance and sensing depth, as indicated by the theory. This task is actually left to the ability of the designer and generally requires an in-depth understanding of the physical phenomenon under observation and of its interaction with the antenna's near-field. Some design guidelines can be however suggested. The shape of the tag antenna has to be fixed according to the object of placement, e.g., a patch antenna is suited to metallic objects while a dipole layout works well for low-loss materials. Then the selection of two or more geometrical degrees of freedom, aimed at the impedance control, has to be related to the physical parameter to be sensed. T-match impedance adapters have demonstrated to be effective in case of dielectric sensing (liquids or powders) while slot-like adapters are more suitable for hosting chemical doping by conductive polymers [25] or carbon nano-tubes to detect gases. In all the cases the design of such devices appear greatly time consuming since the antenna response has to be predicted, for each set of guess geometrical parameters, at each realization of the physical process. More efficient design methodologies are expected to be developed in future research by taking benefit of the most assessed background of ultra wideband antennas and microwave filters since the sensing-tag design is conceptually similar to the frequency-shaping of conventional electromagnetic devices.

\section{REFERENCES}

[1] C. Occhiuzzi, A. Rida, G. Marrocco, and M. Tentzeris, "Rfid passive gas sensor integrating carbon nanotubes," IEEE Trans. Microw. Theory Tech., vol. 59, no. 10, pp. 2674-2684, Oct. 2011.

[2] R. Bhattacharyya, C. Floerkemeier, and S. Sarma, "Rfid tag antenna based sensing: Does your beverage glass need a refill?," in Proc. RFID, Apr. 2010, pp. 126-133.

[3] G. Marrocco, "Pervasive electromagnetics: Sensing paradigms by passive RFID technology," IEEE Wireless Commun., vol. 17, no. 12, pp. 10-17, Dec. 2010.

[4] A. A. Babar, S. Manzari, L. Sydanheimo, A. Z. Elsherbeni, and L. Ukkonen, "Passive UHF RFID tag for heat sensing applications," IEEE Trans. Antennas Propag., vol. 60, no. 9, pp. 4054-4064, Sep. 2012.

[5] S. Merilampi, T. Bjorninen, L. Ukkonen, P. Ruuskanen, and L. Sydanheimo, "Embedded wireless strain sensors based on printed RFID tag," Sens. Rev., vol. 31, no. 1, pp. 32-40, 2011.

[6] S. Johan, X. Zeng, T. Unander, A. Koptyug, and H.-E. Nilsson, "Remote moisture sensing utilizing ordinary RFID tags," Proc. IEEE Sens., pp. 308-311, oct. 2007.

[7] E. Perret, S. Tedjini, and R. Nair, "Design of antennas for UHF RFID tags," Proc. IEEE, vol. 100, pp. 2330-2340, Jul. 2012.

[8] C. Occhiuzzi, G. Contri, and G. Marrocco, "Design of implanted RFID tags for passive sensing of human body: The STENTAG," IEEE Trans. Antennas Propag., vol. 60, no. 7, pp. 3146-3154, Jul. 2012.

[9] C. Occhiuzzi and G. Marrocco, "Electromagnetic optimization of passive RFID sensor nodes," in Proc. EUCAP, Mar. 2012, pp. 53-56.

[10] P. Nikitin and K. Rao, "Theory and measurement of backscattering from RFID tags," IEEE Antennas Propag. Mag., vol. 48, pp. 212-218, Dec. 2006.

[11] A. Pouzin, T. Vuong, S. Tedjini, M. Pouyet, and J. Perdereau, "Bench test for measurement of differential res of UHF RFID tags," Electron. Lett., vol. 46, pp. 590-592, 2010, 15.

[12] R. M. P. V. Nikitin and K. V. S. Rao, "Differential res of RFID tag," Electron. Lett., vol. 43, no. 8, pp. 431-432, 2007.

[13] J. Bolomey, S. Capdevila, L. Jofre, and J. Romeu, "Electromagnetic modeling of RFID-modulated scattering mechanism. Application to tag performance evaluation," Proc. IEEE, vol. 98, pp. 1555-1569, Sep. 2010.

[14] F. Fuschini, C. Piersanti, F. Paolazzi, and G. Falciasecca, "Analytical approach to the backscattering from UHF RFID transponder," IEEE Antennas Wireless Propag. Lett., vol. 7, pp. 33-35, 2008.

[15] G. Marrocco, "Rfid grids: Part I: Electromagnetic theory," IEEE Trans. Antennas Propag., vol. 59, no. 3, pp. 1019-1026, Mar. 2011.

[16] S. Caizzone and G. Marrocco, "Rfid grids: Part ii: Experimentations," IEEE Trans. Antennas Propag., vol. 59, no. 8, pp. 2896-2904, Aug. 2011.

[17] C. Occhiuzzi, S. Caizzone, and G. Marrocco, "Wireless sensing of things by passive RFID technology," IEEE Trans. Antennas Propag., 2012, to be published.

[18] G. Marrocco, E. Di Giampaolo, and R. Aliberti, "Estimation of UHF RFID reading regions in real environments," IEEE Antennas Propag. Mag., vol. 51, no. 12, pp. 44-57, Dec. 2009.

[19] C. Occhiuzzi, C. Paggi, and G. Marrocco, "Passive RFID strainsensor based on meander-line antennas," IEEE Trans. Antennas Propag., vol. 59, pp. 4836-4840, Dec. 2011.

[20] S. Caizzone, C. Occhiuzzi, and G. Marrocco, "Multi-chip RFID antenna integrating shape-memory alloys for detection of thermal thresholds," IEEE Trans. Antennas Propag., vol. 59, no. 7, pp. 2488-2494, Jul. 2011. 
[21] R. Bhattacharyya, C. Floerkemeier, S. Sarma, and D. Deavours, "RFID tag antenna based temperature sensing in the frequency domain," in Proc. RFID, Apr. 2011, pp. 70-77.

[22] K. Rao, P. Nikitin, and S. Lam, "Impedance matching concepts in RFID transponder design," in Proc. IEEE 4th Automatic Identification Adv. Technol. Workshop, Oct. 2005, pp. 39-42.

[23] G. Marrocco, "The art of UHF RFID antenna design: Impedancematching and size-reduction techniques," IEEE Antennas Propag. Mag., vol. 50, pp. 66-79, Feb. 2008.

[24] G. Marrocco and F. Amato, "Self-sensing passive RFID: From theory to tag design and experimentation," in Proc. EuMC, Oct. 2009, pp. 001-004.

[25] S. Manzari, C. Occhiuzzi, S. Nawale, A. Catini, C. Di Natale, and G. Marrocco, "Humidity sensing by polymer-loaded UHF RFID antennas," IEEE Sensors, vol. 12, no. 9, pp. 2851-2858, Sep. 2012.

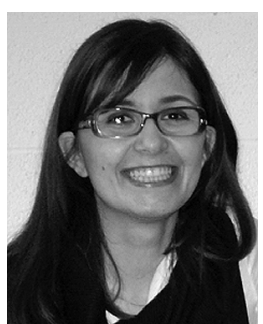

Cecilia Occhiuzzi (M'12) received the M.Sc. degree in medical engineering and the Ph.D. in electromagnetics from the University of Rome "Tor Vergata", in 2008 and 2011, respectively.

Currently, she is a Research Assistant in the University of Rome "Tor Vergata", with interests in wireless health monitoring by means of wearable and implantable radiofrequency identification techniques. In 2008, she was with the School of Engineering, University of Warwick, Warwick, U.K., as a postgraduate student involved with design and implementation of wireless surface acoustic wave (SAW) sensors. In 2010, she was a Visiting Researcher with the Georgia Institute of Technology, Atlanta. Her research was mainly focused on the design of passive RFID sensors for structural health monitoring and gas detection by means of CNT-based tags. She holds two patents on sensor RFID systems. She is co-author of ten Journal Papers, more than twenty International Conference Contributions and a Book Chapter. Finally, she holds two patents on sensor RFID systems.

Dr. Occhiuzzi has been awarded the IEEE APS Doctoral Award in 2011. In 2012 she received the Best Thesis Award at the 2012 RFID Journal Live (Orlando, USA) and, in the same year, the Best Student Paper Award at EUCAP 2012 (Prague). In 2012 She was in the Technical Program Committee of the 2012 RFID-TA, Nice. She is co-founder and CEO of RADIO6ENSE, a spin-off of the University of Rome "Tor Vergata" aiming at designing and developing RFID Sensing and Identification Platforms for Industrial, Civil, Aerospace and Biomedical applications.

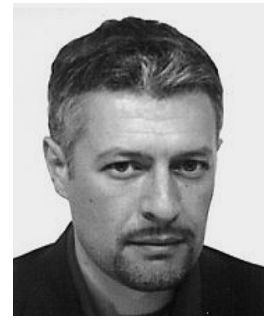

Gaetano Marrocco was born in Teramo, Italy, on August 29, 1969, He received the Laurea degree in electronic engineering (laurea cum laude and academic hons) and the Ph.D. degree in applied electromagnetics from the University of L'Aquila, Italy, in 1994 and 1998, respectively.

Since 1997, he has been a Researcher at the University of Rome "Tor Vergata," Rome, Italy, where he currently teaches Electromagnetic Technology for Wireless Systems and Medical Radio-Systems, manages the Pervasive Electromagnetics Lab and is advisor in the Geo-Information Ph.D. program. In October 2010 he achieved the degree of Associate Professor of Electromagnetic. In 1994, he was at the University of Illinois at Urbana-Champaign as a Postgraduate Student. In 1999, he was a Visiting Researcher at the Imperial College in London, U.K. In 2008 he joined the Ph.D. program of the University of Grenoble (FR). His research is mainly directed to the modeling and design of broadband and ultra wideband (UWB) antennas and arrays as well as of sensor-oriented miniaturized antennas for Biomedicine, Aeronautics and Radiofrequency Identification (RFID).

Prof. Marrocco has been involved in several Space, Avionic, Naval and Vehicular programs of the European Space Agency, NATO, Italian Space Agency, and the Italian Navy about the analysis and the design of non-conventional antennas and systems over platforms. He submitted eight patents on broadband naval antennas and structural arrays, and on sensor RFID systems. Currently, he serves as Associate Editor of the IEEE ANTENNAS AND WIRELESS Propagation LetTers, Vice-Chair of the Italian delegation URSI Commission D: Electronics and Photonics, and as member of Technical Program Committee of IEEE RFID, IEEE IMS and ISABEL. In 2008 he was the General Chairman of the first Italian multidisciplinary scientific workshop on RFID RFIDays-2008: Emerging Technology for Radiofrequency Identification. He was the co-chair of the RFIDays-2010 International Workshop in Finland and Chairman of the Local Committee of the V European Conference on Antennas and Propagation and TCP chair of the 2012 IEEE-RFID TA in Nice, France. Prof. Marrocco is the co-founder of the University spin-off RADIO6ENSE active in the short-range electromagnetic sensing for Aerospace, Healthcare, Internet of Things and Smart Cities. 